\title{
Structural modulation of gut microbiota during alleviation of type 2 diabetes with a Chinese herbal formula
}

\author{
Jia $\mathrm{Xu}^{1,4}$, Fengmei $\mathrm{Lian}^{2,4}$, Linhua Zhao ${ }^{2}$, Yufeng Zhao ${ }^{3}$, Xinyan Chen ${ }^{2}, \mathrm{Xu}$ Zhang ${ }^{1}$,
} Yun Guo ${ }^{2}$, Chenhong Zhang ${ }^{1}$, Qiang Zhou ${ }^{2}$, Zhengsheng Xue ${ }^{1}$, Xiaoyan Pang ${ }^{1}$, Liping Zhao ${ }^{1,3}$ and Xiaolin Tong ${ }^{2}$

${ }^{1}$ State Key Laboratory of Microbial Metabolism, School of Life Sciences and Biotechnology, Shanghai Jiao Tong University, Shanghai, People's Republic of China; ${ }^{2}$ Guang'anmen Hospital, China Academy of Chinese Medical Sciences, Beijing, People's Republic of China and ${ }^{3}$ Ministry of Education Key Laboratory of Systems Biomedicine, Shanghai Center for Systems Biomedicine, Shanghai Jiao Tong University, Shanghai, People's Republic of China

\begin{abstract}
The gut microbiota is hypothesized to have a critical role in metabolic diseases, including type 2 diabetes (T2D). A traditional Chinese herbal formula, Gegen Qinlian Decoction (GQD), can alleviate T2D. To find out whether GQD modulates the composition of the gut microbiota during T2D treatment, 187 T2D patients were randomly allocated to receive high (HD, $n=44)$, moderate (MD, $n=52)$, low dose GQD (LD, $n=50)$ or the placebo $(n=41)$ for 12 weeks in a double-blinded trial. Patients who received the HD or MD demonstrated significant reductions in adjusted mean changes from baseline of fasting blood glucose (FBG) and glycated hemoglobin (HbA1c) compared with the placebo and LD groups. Pyrosequencing of the V3 regions of 16S rRNA genes revealed a dosedependent deviation of gut microbiota in response to GQD treatment. This deviation occurred before significant improvement of T2D symptoms was observed. Redundancy analysis identified 47 GQDenriched species level phylotypes, 17 of which were negatively correlated with FBG and 9 with HbA1c. Real-time quantitative PCR confirmed that GQD significantly enriched Faecalibacterium prausnitzii, which was negatively correlated with FBG, HbA1c and 2-h postprandial blood glucose levels and positively correlated with homeostasis model assessment of $\beta$-cell function. Therefore, these data indicate that structural changes of gut microbiota are induced by Chinese herbal formula GQD. Specifically, GQD treatment may enrich the amounts of beneficial bacteria, such as Faecalibacterium spp. In conclusion, changes in the gut microbiota are associated with the antidiabetic effects of GQD.
\end{abstract}

The ISME Journal (2015) 9, 552-562; doi:10.1038/ismej.2014.177; published online 3 October 2014

\section{Introduction}

Type 2 diabetes (T2D), which is characterized by low-grade inflammation, insulin resistance (Shoelson, 2006) and $\beta$-cell failure (Butler et al., 2003), has become increasingly prevalent worldwide (Xu et al., 2013). The estimated proportion of diabetes among adults is $8.3 \%$ in 2010 , among

Correspondence: L Zhao, Ministry of Education Key Laboratory of Systems Biomedicine, Shanghai Center for Systems Biomedicine, Shanghai Jiao Tong University, Room 3-517, Biology Building, 800 Dongchuan Road, Shanghai 200240, People's Republic of China or X Tong, Guang'anmen Hospital of China Academy of Chinese Medical Sciences, Room 432, Administration Building, 5 Beixiange Street, Xuanwu District, Beijing 100053, China. E-mail: lpzhao@sjtu.edu.cn or xiaolintong66@sina.com ${ }^{4}$ These authors contributed equally to this work.

Received 11 September 2013; revised 18 June 2014; accepted 15 August 2014; published online 3 October 2014 which T2D accounts for at least 90\% (Alberti and Zimmet, 1998; Whiting et al., 2011). This proportion is projected to increase to $9.9 \%$ by 2030 (Whiting et al., 2011). Development of T2D results mostly from obesity, which has low-grade inflammation and insulin resistance (Hotamisligil, 2006).

The gut microbiota may have a vital role in obesity development (Backhed et al., 2004; Collins et al., 2013; Le Chatelier et al., 2013; Zhao, 2013). For example, endotoxin produced by an opportunistic pathogen in the gut, such as Escherichia coli, induced obesity and insulin resistance when a purified form was subcutaneously infused into mice (Cani et al., 2007a). A greater abundance of opportunistic pathogens, such as Betaproteobacteria, was found in the gut of diabetic patients compared with healthy controls (Larsen et al., 2010). A more recent comparative metagenomic analysis of the fecal samples of 171 diabetic patients and 174 
healthy controls showed that diseased samples had lesser abundance of butyrate-producing bacteria, such as Faecalibacterium prausnitzii, but greater abundance of opportunistic pathogens, including Clostridium bolteae and Desulfovibrio sp. (Qin et al., 2012). Another study found that the early onset of high-fat-diet-induced T2D was characterized by an increased bacterial translocation from the intestine towards tissues (Amar et al., 2011). An opportunistic pathogen, Enterobacter cloacae B29, isolated from the gut of a morbidly obese and diabetic patient, induced obesity and insulin resistance in germ-free mice (Fei and Zhao, 2013). Taken together, these studies indicate that a dysbiotic gut microbiota may causatively contribute to obesity and diabetes development, and thus may serve as a potential new target for disease control.

To treat obesity, T2D and other metabolic diseases, several attempts have been made to target the gut microbiota (Cani et al., 2007b, 2009; Park et al., 2013). Berberine, the major pharmacologic component of a Chinese herb Coptis chinensis (HuangLian) originally used to treat bacterial diarrhea (Khin Maung et al., 1985; Rabbani et al., 1987; Tang et al., 2009), showed clinical efficacy in treating diabetes in a multicentered, randomized, double-blinded and placebo-controlled clinical trial (Zhang et al., 2008). The herb C. chinensis has been used in traditional Chinese medicine (TCM) for diarrhea control for nearly 2000 years. Our recent study showed that berberine prevented high-fatdiet-induced obesity and insulin resistance, enriched short-chain fatty acid-producing bacteria, reduced numbers of opportunistic pathogens and alleviated inflammation in Wistar rats (Zhang et al., 2012b).

Drugs that treat bacterial diarrhea, such as berberine, might be useful for T2D treatment because both diseases share a dysbiotic gut microbiota. A standardized berberine-containing Chinese herbal formula, Gegen Qinlian Decoction (GQD), has been a treatment for diarrhea in Shang Han Lun since the East Han Dynasty. Its use was recorded by the prestigious physician Zhongjing Zhang (AD 150219). Subsequently, GQD has been reported to have potentially beneficial effects in the treatment of diabetes in animal trials, as well as in some clinical observations. For example, GQD significantly reduced fasting blood glucose (FBG) and glycated hemoglobin (HbA1c) in streptozotocin (STZ) and high-fat-diet-induced diabetic SD rats, and the serum of SD rats that received GQD enhanced glucose consumption in 3T3-L1 adipocytes (Zhang et al., 2013). T2D patients treated with a high dose of modified GQD two times daily for 3 months showed a reduction in $\mathrm{HbA} 1 \mathrm{c}$ of $1.79 \%$ from the initial level of $9.2 \%$. This decrease was significantly different from that of patients receiving a low-dose treatment (Tong et al., 2011). However, these studies are either animal trials or open, non-placebo-controlled clinical investigations with small sample sizes.
Moreover, the mechanism underlying GQD's impact on glycemic efficacy has barely been elucidated. A recent nuclear magnetic resonance-based plasma metabonomic study revealed that 5 weeks of GQD treatment conspicuously modulated gut microbial metabolism by degradation of choline into methylamines, together with a decrease in FBG and an expansion of islets in STZ and high-fat-diet-induced diabetic rats (Tian et al., 2013). This finding indicates that the gut microbiota might have a pivotal role in the effect GQD has on diabetic subjects. However, there is still a lack of direct evidence showing that GQD can modulate gut microbiota, particularly in humans.

In this study, we conducted a randomized, double-blinded, placebo-controlled clinical trial to evaluate the efficacy and safety of GQD in the treatment of T2D. Furthermore, we examined the structural alterations of gut microbiota in response to GQD treatment intended to alleviate T2D.

\section{Materials and methods}

Study design

The study was a 12-week, randomized, doubleblinded and placebo-controlled clinical trial that included a 2-week washout period. It was approved by the Ethics Committee of Guang'anmen hospital of China Academy of TCM. Participants were recruited by Guang'anmen hospital, Dong Zhimen Hospital affiliated to Beijing TCM University, China-Japan Friendship Hospital or Ji Shui Tan Hospital of Beijing from August 2010 to May 2011. All participants signed informed consent forms before beginning the study. The study was conducted in accordance with the principles of the Declaration of Helsinki.

The inclusion and exclusion criteria of the patients' enrollments can be found in the Supplementary Materials and methods. Using an initial screening, including FBG test and 75-g oral glucose tolerance test, 629 recently diagnosed T2D patients who had not received prior pharmacologic treatment for T2D were recruited into the study. After a 2-week washout period and the review of a series of examinations, 403 patients were excluded for not meeting the inclusion criteria and 2 patients were excluded for other reasons. The remaining 224 patients were randomly assigned to four groups of 56 patients. Each group received one of the following treatments: high (HD), moderate (MD) or low dose (LD) GQD, or the placebo for 12 weeks. Randomization was performed centrally and was concealed and stratified in blocks of eight by the PROC PLAN process using the SAS software (SAS Institute Inc., Cary, NC, USA). After the study was completed, a total of 187 patients were included for the final analysis by the verification of data examination committee (Supplementary Figure 1). 
Drug administration

The TCM formula in our study was GQD, composed of four herbs, namely: Gegen (Radix Puerariae), Huangqin (Radix Scutellariae), Huanglian (Rhizoma Coptidis) and Gancao (Honey-fried Licorice Root) (Supplementary Figure 2a). Supplementary Table 1 lists the amount of each herb in one unit of GQD formula in each group. Herbs were all provided and quality controlled by Beijing Shuangqiaoyanjing Chinese herb manufacturer (Supplementary Materials and methods). The TCM intervention and placebo were given as decoction; these were prepared by Beijing Jiulong Pharmaceutical Factory according to a standard production process (Supplementary Materials and methods). Each unit of GQD formula or placebo yielded $300 \mathrm{ml}$ of decoction. Each patient orally took $150 \mathrm{ml}$ of the decoction two times daily for 12 weeks. All of the drugs and decoctions were quality controlled throughout the trial, and the placebo decoction was prepared by the same standardized process (Supplementary Materials and methods).

\section{Study evaluation and outcomes}

The following primary efficacy outcomes were used: changes in HbA1c, FBG and 2-h postprandial blood glucose (2h-PBG) levels. Secondary efficacy outcomes included changes in serum insulin, lipids levels and body mass index. Study assessments were performed at $0,4,8$ and 12 weeks. Measurements of FBG, 2h-PBG, body mass index, waist circumference and hip circumference were taken at 0, 4, 8 and 12 weeks. Serum HbA1c, insulin, total cholesterol, triglycerides, high-density lipoprotein cholesterol and low-density lipoprotein cholesterol measurements were performed at weeks 0 and 12 . Fecal samples were collected every 4 weeks until the end of trial for gut microbiota analysis.

\section{Clinical and biochemical measurements}

Biochemical measurements of glucose, serum lipids, HbA1c and insulin were performed in a central laboratory (Guang'anmen Hospital of China Academy of TCM, Beijing, China). Glucose, serum total cholesterol, triglycerides, high-density lipoprotein cholesterol and low-density lipoprotein cholesterol were measured by enzymatic methods (Olympus AU2700; Olympus Co. Ltd., Tokyo, Japan). HbA1c was measured by high-performance liquid chromatography using a variant hemoglobin HbA1c assay (ADAMS A1c HA-8160; Arkray Inc., Kyoto, Japan). Serum insulin was measured using a double-antibody RIA (ADVIA Centaur; Bayer Diagnostics, Leverkusen, Germany). ELISA Kits were used to measure plasma orosomucoid (Assaypro, St Charles, MO, USA), adiponectin (R\&D System, Minneapolis, MN, USA), tumor necrosis factor- $\alpha$ (R\&D System) and serum amyloid A protein (Invitrogen, Carlsbad, CA, USA) levels.
Fecal DNA extraction and pyrosequencing

Genomic DNA of each fecal sample was extracted by a InviMag Stool DNA Kit (Invitek, Berlin, Germany) combined with bead beating as previously published (Zhang et al., 2012b). The extracted genomic DNA was used as the template to amplify the V3 region of $16 \mathrm{~S}$ rRNA genes. PCR reactions, pyrosequencing of the PCR amplicons and quality control of raw data were performed as described previously with minor modification (Zhang et al., 2009; Wang et al., 2011).

\section{Bioinformatics and multivariate statistics}

High-quality sequence alignments were performed using NAST. Sequence clustering by CD-hit and OUT delineation by DOTUR were performed as described previously (Zhang et al., 2012a, b). The representative sequences of operational taxonomy units (OTUs) with their relative abundance were used to calculate rarefaction analysis and Shannon diversity index by QIIME (Caporaso et al., 2010). In addition, the representative sequences were inserted into a pre-established phylogenetic tree of the full-length $16 \mathrm{~S}$ rRNA gene sequences in ARB (Ludwig et al., 2004). Then, the phylogenetic tree and the relative abundance table of representative sequences of OTUs were used for UniFrac principal coordinate analysis (PCoA) (Lozupone and Knight, 2005). The statistical significance between different groups was assessed by multivariate analysis of variance in MATLAB 2010b (The MathWorks Inc., Natick, MA, USA). Redundancy analysis was performed using CANOCO for Windows 4.5 (Microcomputer Power, Ithaca, NY, USA) according to the manufacturer's instructions (Braak and Smilauer, 2002). Statistical significance was assessed by MCPP with 499 random permutations under the full model. Ribosomal Database Project Classifier was used to assess the amounts of different genera by taxonomic assignment of all sequences.

\section{Real-time quantitative PCR of F. prausnitzii}

Real-time quantitative PCR (q-PCR) was used to determine the amounts of total bacteria and $F$. prausnitzii through detection of $16 \mathrm{~S}$ rRNA genes. A set of universal primers was used to amplify a conserved 16S rDNA sequence in all bacteria as shown before (Wang et al., 2011). A set of specific primers was used to amplify a conserved $16 \mathrm{~S}$ rDNA sequence in $F$. prausnitzii and the q-PCR reaction system and the program was described before (Balamurugan et al., 2008). A plasmid containing a $F$. prausnitzii full-length $16 \mathrm{~S}$ rDNA from a previous study (Shen et al., 2006) was prepared using the EZNA Plasmid Mini Kit I (OMEGA, Doraville, GA, USA) and diluted from $1 \times 10^{3}$ to $1 \times 10^{9}$ (copies $\mu \mathrm{l}^{-1}$ ) to construct a standard curve for the detection of $F$. prausnitzii. We selected reactions with efficiencies ranging from 0.90 to 1.05 for 
further analysis. Standard and quantified samples were performed in triplicate. PCR reactions were performed using iQ SYBR Green SuperMix (BioRad, Richmond, CA, USA) on a MasterCycler ep Realplex 4s (Eppendorf, Hamburg, Germany).

Spearman's correlation coefficient $(R)$ and $P$-value were used to compare the amounts of $F$. prausnitzii measured by q-PCR and pyrosequencing. This coefficient was also used to evaluate the relationship between $F$. prausnitzii and clinical parameters using MATLAB 2010b.

\section{Results}

The major components of $G Q D$ decoction

There were four major categories of compounds in the GQD decoction. Flavones (baicalin, puerarin, wogonoside, daidzin, liquiritin, baicalein and wogonin), alkaloids (berberine, coptisine, palmatine and jatrorrhizine) and triterpenoid sapnins (glycyrrhizin) were detected in the decoction, among which baicalin, puerarin and berberine were the major components (Supplementary Table 2). The chemical structures of these 12 components are shown in the Supplementary Figure 2c. Carbohydrates (starch, sucrose, reducing sugar and soluble dietary fiber) were also detected. Insoluble dietary fiber was undetectable in GQD decoction (Supplementary Table 3).

\section{GQD significantly improved glycemic control in T2D} patients

In our 12-week, randomized, double-blinded, placebo-controlled clinical trial (Supplementary Figure 1), the data of 187 participants were analyzed as shown in Supplementary Table 4. The baseline variables were not significantly different among the four groups. After 12 weeks of treatment, GQD significantly improved glycemic control in T2D patients. The HD and MD groups, when compared with the placebo and LD groups, showed significant reductions in adjusted mean changes from baseline of FBG (-1.46 \pm 0.23 and $-1.09 \pm 0.21$ vs $-0.16 \pm 0.22$ and $-0.24 \pm 0.24 \mathrm{mmoll}^{-1} ; P<0.001$ for HD vs LD and placebo; $P<0.01$ for MD vs $\mathrm{LD}$ and placebo). Similarly, the HD and MD groups showed significantly reduced HbA1c $(-0.88 \pm 0.14$ and $-0.75 \pm 0.13$ vs $-0.35 \pm 0.13$ and $-0.36 \pm 0.15 \%$; $P<0.01$ for $\mathrm{HD}$ vs $\mathrm{LD} ; P<0.05$ for $\mathrm{HD}$ vs placebo; $P<0.05$, MD vs $\mathrm{LD}$ and placebo) (Figures $1 \mathrm{a}$ and $\mathrm{b}$ ). A decrease in the mean change of $2 \mathrm{~h}-\mathrm{PBG}$ from baseline was also observed in the treated groups, although not reaching significant level. (Supplementary Figure 3). In addition, ANCOVA analysis showed that HOMA- $\beta$ was significantly improved by HD GQD treatment compared with the placebo and LD groups (Figure 1c).

Plasma orosomucoid was significantly reduced by HD GQD treatment $(P=0.023)$ (Supplementary Figure $4 \mathrm{a}$ ) and the HD group showed a significant reduction $(P=0.034)$ in mean change from baseline of plasma orosomucoid compared with the LD group (Supplementary Figure 4b). No significant difference was observed in plasma adiponectin, tumor necrosis factor- $\alpha$ or serum amyloid A among the four groups (Supplementary Figures 5a-c). Finally, no drug-related serious adverse events occurred in this study.

\section{Overall structural modulation of gut microbiota after GQD treatment}

First, we used a bar-coded pyrosequencing run to analyze the structural changes of gut microbiota in the four groups before and after GQD treatment. In total, 483304 usable raw sequences (34753 unique sequences) and 3222 OTUs were obtained from 235 samples with an average of $2057 \pm 396$ per sample. Rarefaction and Shannon diversity curves revealed that, although no rarefaction curves plateaued with the current sequencing, most of the diversity had already been captured (Supplementary Figure 6). Weighted and unweighted UniFrac PCoA analysis revealed that gut microbiota structure of the treated groups showed a dose-dependent deviation
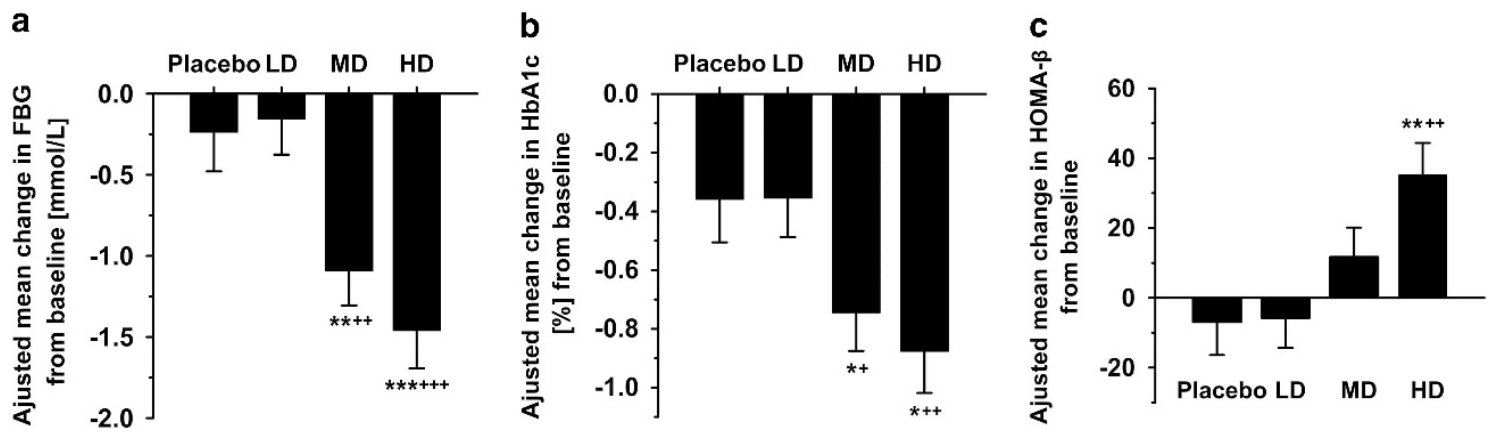

Figure 1 GQD significantly improved glycemic control and HOMA- $\beta$ in T2D patients. (a) Change in FBG, (b) change in HbA1c and (c) change in HOMA- $\beta$. Placebo $(n=41)$, LD $(n=50), \mathrm{MD}(n=52)$ and $\mathrm{HD}(n=44)$. Data are presented as mean \pm S.E.M. ${ }^{*} P<0.05$, ${ }^{*} P<0.01$ and ${ }^{* *} P<0.001$ vs placebo using ANCOVA; ${ }^{+} P<0.05,{ }^{++} P<0.01$ and ${ }^{++}{ }^{+} P<0.001$ vs LD using ANCOVA. 
from the baseline structure, with the HD group reaching significant level in multivariate analysis of variance test (Figures 2a and b and Supplementary Figures 7a and b).

To monitor the dynamic changes of gut microbiota during GQD treatment, we analyzed the fecal samples collected at weeks $0,4,8$ and 12 in HD and placebo groups with a second pyrosequencing run. In total, we generated 680774 usable raw sequences (37498 unique sequences) and 4251 OTUs from 288 samples with an average of $2364 \pm 443$ per sample (one sample was excluded in later analysis because only 81 reads were obtained). Rarefaction and Shannon diversity curves revealed that most of the diversity had already been captured (Supplementary Figure 8). UniFrac PCoA and PCA showed that after 4 weeks of treatment, the gut microbiota structure of the HD group had already significantly diverged from that of its baseline and of the placebo group (Figures $3 \mathrm{a}$ and b; Supplementary Figure 9). At that same 4-week analysis, 2h-PBG was significantly reduced, but FBG did not reach a significant level in treated groups (Supplementary Figure 10). As the treatment
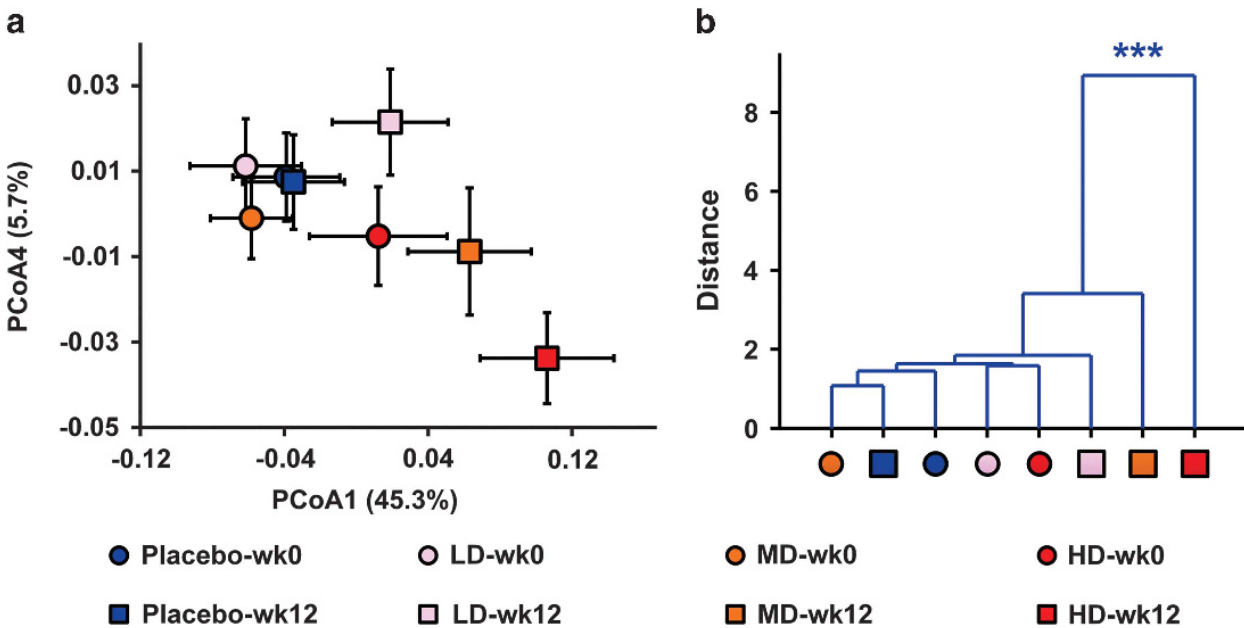

Figure 2 Dose-dependent alterations of the gut microbiota in T2D patients treated with different doses of GQD at weeks 0 and 12. (a) Weighted Unifrac PCoA of gut microbiota based on the OUT data from the first pyrosequencing run. (b) Clustering of gut microbiota based on mahalanobis distances calculated with multivariate analysis of variance (MANOVA). Each point represents the mean principal coordinate (PC) score of all patients in a group at one time point, and the error bar represents the s.e.m. The sample number $(n)$ at week 0 : placebo $=30, \mathrm{LD}=28, \mathrm{MD}=32$ and $\mathrm{HD}=28$. The sample number $(n)$ at week 12 : placebo $=30, \mathrm{LD}=28, \mathrm{MD}=32$ and $\mathrm{HD}=28$. $* * * P<0.0001$.

a

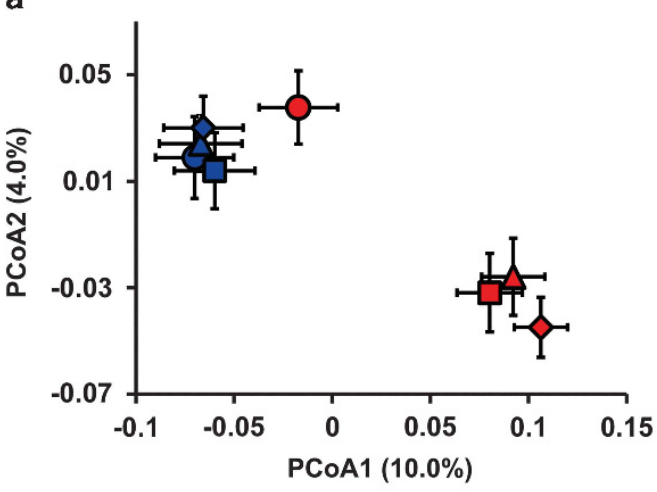

O Placebo-wk0

HD-wk0
$\Delta$ Placebo-wk4

$\Delta$ HD-wk4 b

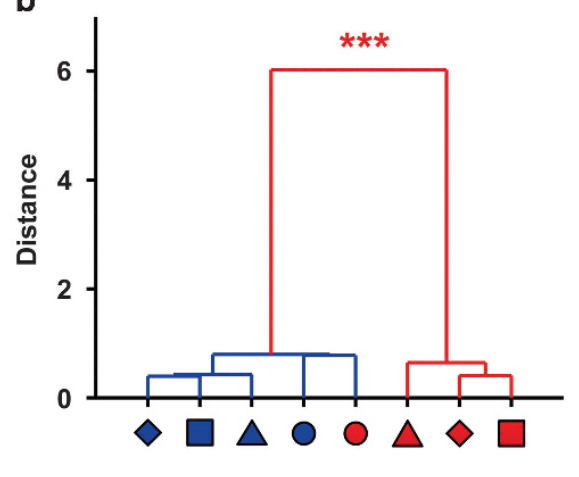

Placebo-wk8

$\diamond$ HD-wk8
Placebo-wk12

HD-wk12

Figure 3 Trajectory of the gut microbiota in T2D patients treated with HD GQD and placebo at weeks 0, 4, 8 and 12. (a) Unweighted Unifrac PCoA of gut microbiota based on the OUT data from the second pyrosequencing run. (b) Clustering of gut microbiota based on mahalanobis distances calculated with multivariate analysis of variance (MANOVA). Each point represents the mean principal coordinate (PC) score of all patients in a group at one time point, and the error bar represents the s.e.m. Placebo: $n=36$; HD: $n=36$. $* * * P<0.0001$. 
progressed, the gut microbiota made no additional changes (Figures $3 \mathrm{a}$ and $\mathrm{b}$ and Supplementary Figure 9), but the FBG and 2h-PBG continued to improve until the end of the study (Supplementary Figure 10).

Key phylotypes responding to the GQD treatment in T2D patients

By using redundancy analysis, we identified 146 key variables that responded to the GQD treatment (Figure 4). Forty-seven OTUs were increased by GQD and 99 were decreased. Among the 47 OTUs enriched by GQD, 17 OTUs showed a significant negative correlation with FBG; the OTUs belong to Faecalibacterium $(n=4)$, Gemmiger $(n=4)$, Bifidobacterium $(n=3)$, Lachnospiracea_incertae_ sedis $(n=2)$ and Escherichia $(n=1)$. Nine OTUs showed a significant negative correlation with HbA1c; two of which were from Faecalibacterium, two from Gemmiger, one from Bifidobacterium, one from Parasutterella and one from Escherichia (Figure 5 and Supplementary Tables 5 and 6). Among the 99 OTUs decreased by GQD treatment, 22 OTUs showed a significant positive correlation with FBG: the OTUs belong to Alistipes $(n=6)$, Odoribacter $(n=2), \quad$ Parabacteroides $(n=2)$, Bacteroides $(n=2)$, Pseudobutyrivibrio $(n=2)$ and one OTU to each of the following genera: Butyricimonas, Barnesiella, Oscillibacter and ClostridiumXIVa. Fourteen OTUs showed a significant positive correlation with HbA1c, four of which were from Alistipes, two from Odoribacter, one from each of the following genera: Parabacteroides, Bacteroides, Pseudobutyrivibrio, Butyricimonas, Barnesiella, Oscillibacter and ClostridiumXlVa (Figure 5 and Supplementary Tables 5 and 6).
Additionally, taxon-based analysis at the genus level showed that the relative abundance of Faecalibacterium, Gemmiger, Bifidobacterium and Lachnospiracea_incertae_sedis was significantly higher after HD GQD treatment, whereas Alistipes, Parabacteroides and Pseudobutyrivibrio were significantly decreased (relative abundance $>1 \%$ and $P<0.01$ ) (Supplementary Figure 11).

\section{Quantification of F. prausnitzii and its association} with glycemic parameters

One previous study showed that $F$. prausnitzii is more abundant in the gut of healthy people compared with T2D patients (Qin et al., 2012). In our study, pyrosequencing results indicated that Faecalibacterium was substantially enriched after GQD treatment. To confirm the pyrosequencing results, we quantified the relative abundance of F. prausnitzii, the prominent species of the Faecalibacterium genus, by q-PCR. All three doses of GQD treatment significantly enriched $F$. prausnitzii compared with baseline $(P=0.004$ for the HD group, $P=0.024$ for the MD group, $P=0.011$ for the LD group, $P=0.217$ for the placebo group) (Figure 6a). Particularly, the relative abundance of this bacterium was significantly higher in HD group than in the other three groups after 12 weeks of treatment $(P=0.0002$, HD vs placebo; $P=0.0435$, HD vs MD; $P=0.0120$, HD vs LD), while no significant difference was observed at baseline (Figure 6b). The relative abundance of Faecalibacterium by q-PCR and pyrosequencing showed a high significant correlation with each other, indicating that the differences of Faecalibacterium among four groups found by pyrosequencing is reliable (Supplementary Figure 12). Moreover, the relative

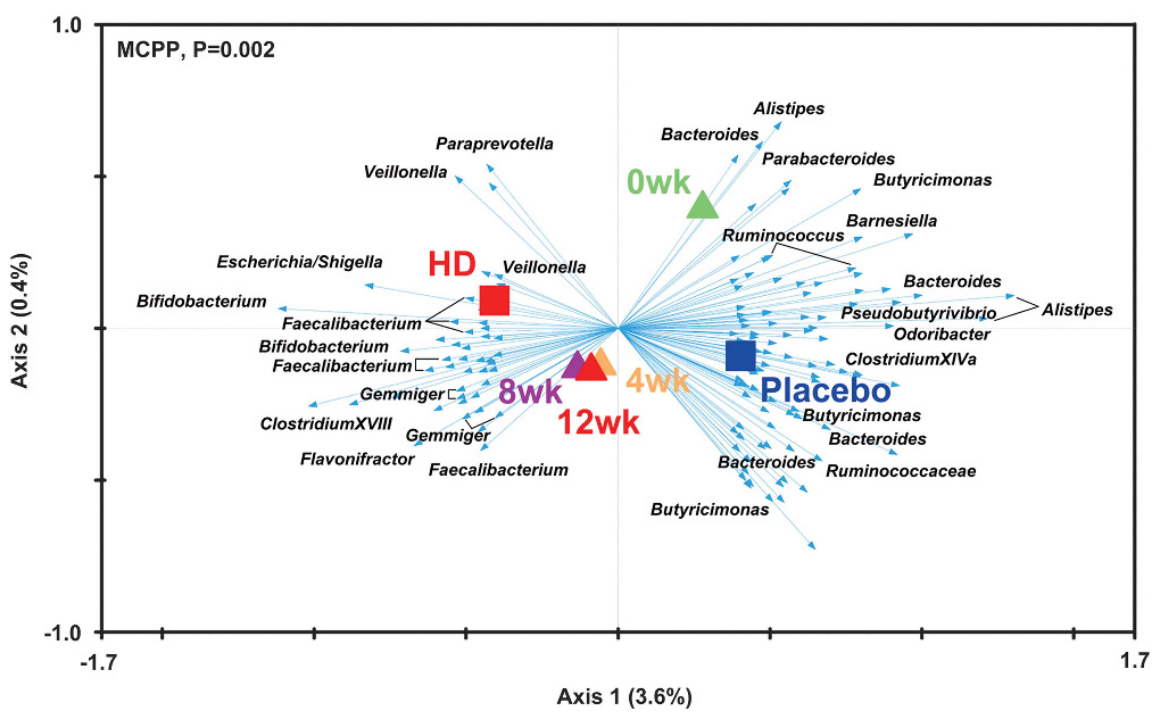

Figure 4 Biplot of redundancy analysis (RDA) of the microbiota composition responding to GQD therapy in T2D patients. The type of treatment and time were used as environmental variables. Responding OTUs that explained more than $4 \%$ of the variability of the samples are indicated by blue arrows. Top-left, $P$-value was obtained by Monte Carlo permutation procedure (MCPP). 


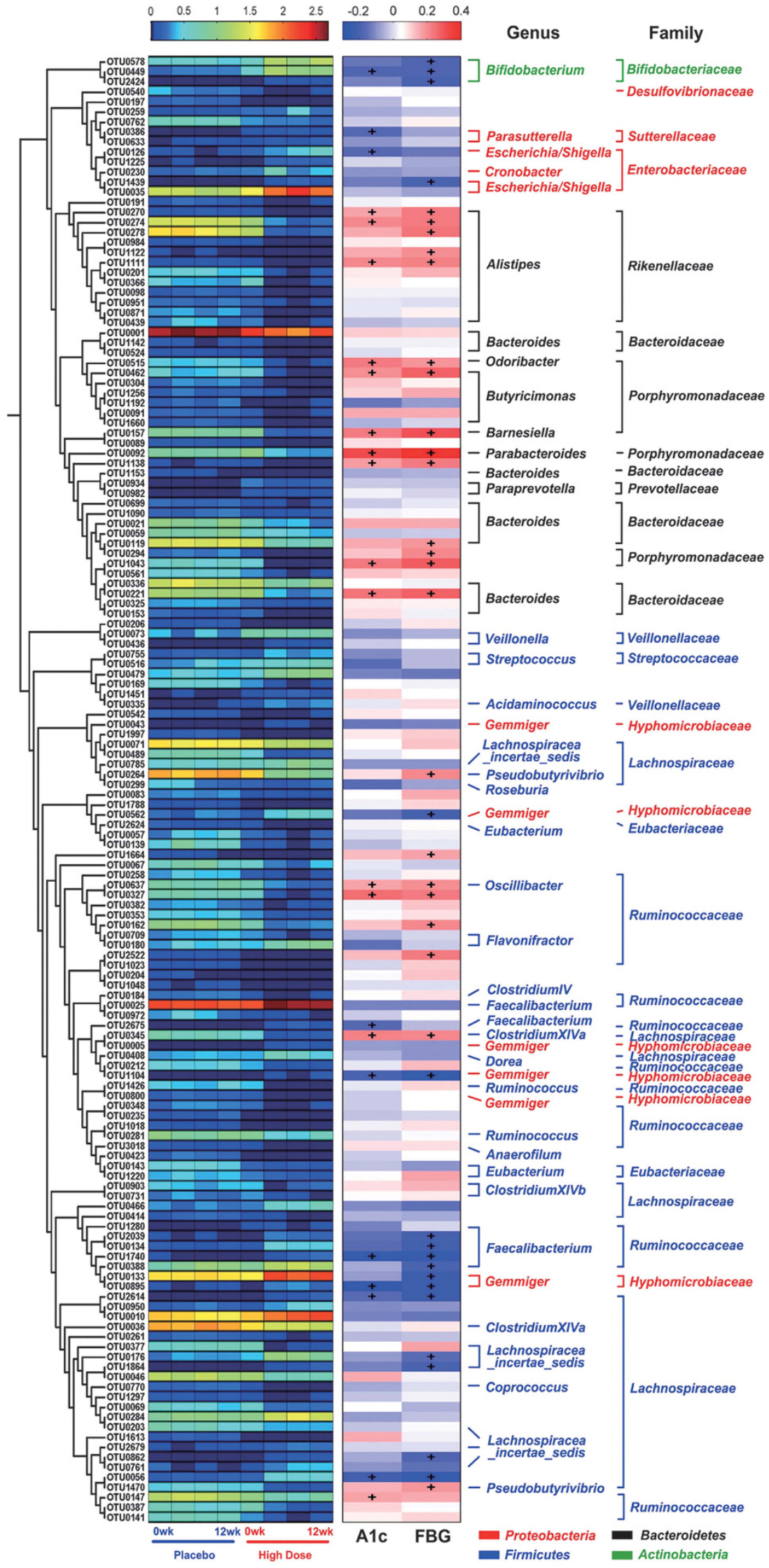


abundance of this bacterium negatively correlated with HbA1c, FBG and 2h-PBG, and positively correlated with HOMA- $\beta$ by Spearman's correlation coefficient (Supplementary Table 7).

\section{Discussion}

To our knowledge, this study is the first registered clinical trial to evaluate the dose-dependent efficacy and safety of a Chinese herbal decoction following a randomized, double-blinded and placebo-controlled design. In our study, GQD treatment provided clinically meaningful and dose-dependent reductions in FBG and HbA1c compared with placebo. HOMA- $\beta$ was also significantly improved by HD GQD treatment. Compared with those in T2D patients treated with berberine in a previously published clinical trial, the HD group in our study showed similar reductions of FBG and HbA1c; however, we did not observe an improvement of dyslipidemia, which had been significantly ameliorated by berberine in the other study (Zhang et al., 2008). In addition, we found a slight alleviation of inflammation, indicated by the reduction of plasma orosomucoid in the HD group. This finding was consistent with a previous nuclear magnetic resonance-based plasma metabonomic study in T2D rats treated with GQD (Tian et al., 2013). These data show that the Chinese herbal formula is effective for glycemic control, making it a promising candidate for diabetes management.

Concomitant with the improved glucose homeostasis, we observed an altered microbial composition induced by GQD. In other studies, diet (Cani et al., 2008; Turnbaugh et al., 2008; Xiao et al.,
2013), drugs (Zhang et al., 2012b; Shin et al., 2013), prebiotics (Cani et al., 2007b, 2009) and probiotics (Park et al., 2013) have been used to modulate the gut microbiota in metabolic diseases, for example, the increase of Akkermansia spp. by metformin in the improvement of glucose homeostasis in high-fatdiet-induced obese mice (Shin et al., 2013). Berberine, the major pharmacologic component of a Chinese herb C. chinensis (Huang-Lian), has been shown to enrich short-chain fatty acid producers in parallel with the prevention of obesity and insulin resistance in rats (Zhang et al., 2012b). C. chinensis is also a major component of GQD. However, no gut microbiota modulation by berberine or Chinese herbs has been reported in humans. To our knowledge, this study is the first direct evidence in humans that TCMs can modulate the structure of the gut microbiota.

Consistent with the dose-dependent manner of T2D amelioration, GQD also exerted a dose-dependent modulation on the gut microbiota, suggesting a strong association between the modulation of gut microbiota and T2D alleviation. Dose-dependent modulation of gut microbiota was also reported in prebiotic studies. For example, an stepwise increase of Bifidobacteria was observed by q-PCR when nine healthy adults received successively increased dosage levels of galactooligosaccharides (Davis et al., 2010). Our study is the first example that dose-dependent modulation of the gut microbiota was concomitant with a dose-dependent alleviation of T2D in humans. Moreover, the gut microbiota alterations occurred before significant improvement of T2D symptoms, supporting the hypothesis that changes in the gut microbiota induced by GQD might contribute to improved glucose homeostasis
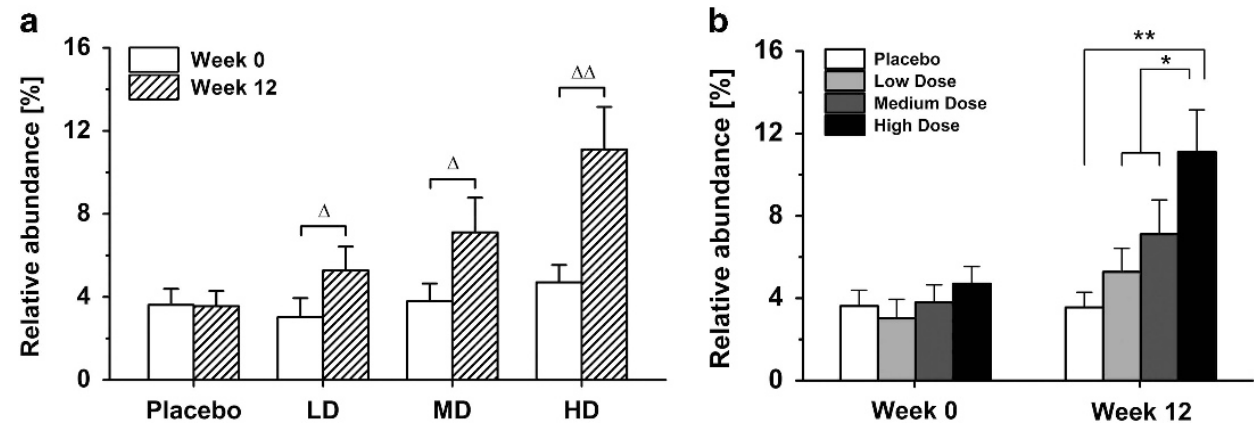

Figure 6 Relative abundance of $F$. prausnitzii as quantified by q-PCR. (a) The impact of different treatments on the relative abundance of $F$. prausnitzii before and after treatment. The sample number $(n)$ for each group: placebo $=21, L D=19, M D=19$ and $H D=20$. $(\mathbf{b})$ The differences of $F$. prausnitzii among four groups at week 12. The sample number $(n)$ for each group at week 0 : placebo $=31$, $L D=23$, $\mathrm{MD}=26$ and $\mathrm{HD}=29$. The sample number for each group at week 12: placebo $=26, \mathrm{LD}=25, \mathrm{MD}=24$ and $\mathrm{HD}=28 .{ }^{\Delta} P<0.05,{ }^{\Delta} P<0.05$ vs before treatment using Wilcoxon's signed rank test. ${ }^{*} P<0.05$ and ${ }^{*} P<0.01$. Placebo, LD, or MD vs HD using Mann-Whitney test. Data are represented as mean \pm s.e.m.

Figure 5 Heat map of redundancy analysis (RDA)-identified key OTUs responding to GQD treatment and Spearman's correlation between identified OTUs and HbA1c or FBG. The color of the spots in the left panel represents the mean relative abundance (normalized and log-transformed) of the OTU in each group. The OTUs are organized according to their phylogenetic positions. The color of the spots in the right panel represents $R$-value of Spearman's correlation between the OTU and HbA1c or FBG. The family and genus names of the OTUs are shown on the right. ${ }^{+} P<0.05$. 
in T2D patients rather than a mere consequence after the symptoms have been alleviated.

Several putative beneficial genera that responded to GQD treatment were identified using redundancy analysis, including Faecalibacterium, Bifidobacterium and Gemmiger. Faecalibacterium, a butyrateproducing bacterial group (Duncan et al., 2002), which was profoundly enriched by GQD in our study, shows anti-inflammatory effects partly through reducing colonic cytokine synthesis and increasing anti-inflammatory cytokine secretion (Sokol et al., 2008). Diabetic obese patients have lower abundance of $F$. prausnitzii compared with non-diabetic obese patients, which demonstrates a negative correlation with inflammatory cytokines C-reactive protein and interleukin-6 (Furet et al., 2010). Another study also showed that the gut of T2D patients is characterized by a reduction of $F$. prausnitzii compared with that of healthy people (Qin et al., 2012). Furthermore, F. prausnitzii has been shown to be a functionally important phylotype because it was associated with eight urinary metabolites (Li et al., 2008). In our study, the relative abundance of $F$. prausnitzii was negatively correlated with $\mathrm{HbA1c}$, FBG and 2h-PBG, and positively correlated with HOMA- $\beta$, suggesting that $F$. prausnitzii might be a pivotal phylotype associated with the improvement of T2D.

Two other genera, Bifidobacterium and Gemmiger, which are also reported to confer beneficial effects (Gossling and Moore, 1975; Sokol et al., 2008; Fukuda et al., 2011), were significantly enriched by GQD in T2D patients. A selective increase of Bifidobacteria induced by a prebiotic (namely oligofructose) improved gut permeability and inflammation in ob/ob mice (Cani et al., 2009). In addition, several genera were significantly inhibited by GQD, such as Alistipes and Odoribacter. Higher abundance of several taxa of the genus Alistipes were associated with greater frequency of abdominal pain in irritable bowel syndrome pediatric patients (Saulnier et al., 2011). One example from this genus, Alistipes putredinis, was isolated from inflamed and non-inflamed intestinal tissues of children with suspected acute appendicitis (Rautio et al., 2000, 2003). Alistipes and Odoribacter were significantly increased in grid floor stress-induced BALB/c mice (Bangsgaard Bendtsen et al., 2012). These results suggest that the enrichment of beneficial bacteria, particularly Faecalibacterium spp., and reduction of pathogen-like bacteria might be involved in the amelioration of T2D by GQD.

TCM formula, a form of polypharmacy, has been developed and advocated for use in the treatment of many diseases for over 2500 years in China. However, the complexities and unknown mechanisms of TCMs prevent the active chemical components from being identified. Our study suggests that gut microbiota might be involved in the effect of a widely used TCM formula, GQD. This opens an avenue for identifying chemical components in
TCMs, which can modulate gut microbiota structure as a potential mechanism for disease alleviation. Baicalin, puerarin and berberine were identified as the three most abundant chemical components in GQD. Baicalin, which demonstrated antioxidant properties together with the glucose-lowering effects in STZ-induced diabetic rats (Waisundara et al., 2009), showed dose-dependent synergic effects with $\beta$-lactam antibiotics against methicillin-resistant Staphylococcus aureus and other $\beta$-lactam-resistant strains of $S$. aureus in vitro (Liu et al., 2000). Puerarin can dose-dependently increase glucose utilization in STZ-induced diabetic rats (Hsu et al., 2003). However, it showed poor absorption into the bloodstream after oral administration in vivo (Luo et al., 2011) and was mainly excreted via feces in its intact form (Zhu et al., 1979). It is possible that puerarin could have an impact on gut microbiota, but no study about this has been reported yet. Berberine showed significant glucose-lowering effects in a multicentered, randomized, doubleblinded and placebo-controlled clinical trial (Zhang et al., 2008). Additionally, berberine has been reported to modulate gut microbiota during prevention of high-fat-diet-induced obesity and insulin resistance in rats (Zhang et al., 2012b), implying that berberine could be one of the major active ingredients in GQD that modulated the gut microbiota in our study. The carbohydrate components of GQD likely exerted few effects on gut microbiota because insoluble dietary fiber was undetectable and soluble dietary fiber was very low. Additionally, these components showed no dosage differences across the four decoctions. Other carbohydrates, such as starch, were easily digested and absorbed before they reached large intestine. These results indicate that modulation of gut microbiota by chemical components, such as berberine, might be involved in improving glucose homeostasis by GQD, suggesting that TCMs may serve as a new source for drug leads in gut microbiota-targeted diabetes management.

In conclusion, our study suggests that structural alterations of gut microbiota, induced by Chinese herbal formula GQD, are associated with the antidiabetic effects of GQD. In particular, this treatment enriched the number of beneficial bacteria, such as Faecalibacterium spp. in the gut. Although it is still unclear whether changes of gut microbiota by GQD directly contribute to the improvement of glucose homeostasis, our clinical study provides circumstantial evidence that gut microbiota might be involved.

\section{Conflict of Interest}

The authors declare no conflict of interest.

\section{Acknowledgements}

This work was supported by Project 2010CB530601 of the National Basic Research Program of China (973 Program), 
Project 81273720 of the National Natural Science Foundation of China (NSFC) and Key program 31330005 of the NSFC.

\section{References}

Alberti KG, Zimmet PZ. (1998). Definition, diagnosis and classification of diabetes mellitus and its complications. Part 1: diagnosis and classification of diabetes mellitus provisional report of a WHO consultation. Diabet Med 15: 539-553.

Amar J, Chabo C, Waget A, Klopp P, Vachoux C, Bermúdez-Humarán LG et al. (2011). Intestinal mucosal adherence and translocation of commensal bacteria at the early onset of type 2 diabetes: molecular mechanisms and probiotic treatment. EMBO Mol Med 3: 559-572.

Backhed F, Ding H, Wang T, Hooper LV, Koh GY, Nagy A et al. (2004). The gut microbiota as an environmental factor that regulates fat storage. Proc Natl Acad Sci USA 101: 15718-15723.

Balamurugan R, Janardhan HP, George S, Chittaranjan SP, Ramakrishna BS. (2008). Bacterial succession in the colon during childhood and adolescence: molecular studies in a southern Indian village. Am J Clin Nutr 88: 1643-1647.

Bangsgaard Bendtsen KM, Krych L, Sorensen DB, Pang W, Nielsen DS, Josefsen K et al. (2012). Gut microbiota composition is correlated to grid floor induced stress and behavior in the BALB/c mouse. PLoS One 7: e46231.

Braak CT, Smilauer P. (2002). CANOCO Reference Manual and CanoDraw for Windows User's Guide: Software for Canonical Community Ordination (version 4.5). Microcomputer Power: Ithaca, New York.

Butler AE, Janson J, Bonner-Weir S, Ritzel R, Rizza RA, Butler PC. (2003). Beta-cell deficit and increased beta-cell apoptosis in humans with type 2 diabetes. Diabetes 52: 102-110.

Cani PD, Amar J, Iglesias MA, Poggi M, Knauf C, Bastelica D et al. (2007a). Metabolic endotoxemia initiates obesity and insulin resistance. Diabetes 56: 1761-1772.

Cani PD, Neyrinck AM, Fava F, Knauf C, Burcelin RG, Tuohy KM et al. (2007b). Selective increases of bifidobacteria in gut microflora improve high-fatdiet-induced diabetes in mice through a mechanism associated with endotoxaemia. Diabetologia 50: 2374-2383.

Cani PD, Bibiloni R, Knauf C, Waget A, Neyrinck AM, Delzenne NM et al. (2008). Changes in gut microbiota control metabolic endotoxemia-induced inflammation in high-fat diet-induced obesity and diabetes in mice. Diabetes 57: 1470-1481.

Cani PD, Possemiers S, Van de Wiele T, Guiot Y, Everard A, Rottier O et al. (2009). Changes in gut microbiota control inflammation in obese mice through a mechanism involving GLP-2-driven improvement of gut permeability. Gut 58: 1091-1103.

Caporaso JG, Kuczynski J, Stombaugh J, Bittinger K, Bushman FD, Costello EK et al. (2010). QIIME allows analysis of high-throughput community sequencing data. Nat Methods 7: 335-336.

Collins SM, Kassam Z, Bercik P. (2013). The adoptive transfer of behavioral phenotype via the intestinal microbiota: experimental evidence and clinical implications. Curr Opin Microbiol 16: 240-245.

Davis LM, Martinez I, Walter J, Hutkins R. (2010). A dose dependent impact of prebiotic galactooligosaccharides on the intestinal microbiota of healthy adults. Int J Food Microbiol 144: 285-292.

Duncan SH, Hold GL, Harmsen HJ, Stewart CS, Flint HJ. (2002). Growth requirements and fermentation products of Fusobacterium prausnitzii, and a proposal to reclassify it as Faecalibacterium prausnitzii gen. nov., comb. nov. Int J Syst Evol Microbiol 52: 2141-2146.

Fei N, Zhao L. (2013). An opportunistic pathogen isolated from the gut of an obese human causes obesity in germfree mice. ISME J 7: 880-884.

Fukuda S, Toh H, Hase K, Oshima K, Nakanishi Y, Yoshimura K et al. (2011). Bifidobacteria can protect from enteropathogenic infection through production of acetate. Nature 469: 543-547.

Furet JP, Kong LC, Tap J, Poitou C, Basdevant A, Bouillot JL et al. (2010). Differential adaptation of human gut microbiota to bariatric surgery-induced weight loss: links with metabolic and low-grade inflammation markers. Diabetes 59: 3049-3057.

Gossling J, Moore WEC. (1975). Gemmiger formicilis, n. gen., n. sp., an anaerobic budding bacterium from intestines. Int J Syst Bacteriol 25: 202-207.

Hotamisligil GS. (2006). Inflammation and metabolic disorders. Nature 444: 860-867.

Hsu FL, Liu IM, Kuo DH, Chen WC, Su HC, Cheng JT. (2003). Antihyperglycemic effect of puerarin in streptozotocin-induced diabetic rats. J Nat Prod 66: 788-792.

Khin Maung U, Myo K, Nyunt Nyunt W, Aye K, Tin U. (1985). Clinical trial of berberine in acute watery diarrhoea. BMJ (Clin Res Ed) 291: 1601-1605.

Larsen N, Vogensen FK, van den Berg FWJ, Nielsen DS, Andreasen AS, Pedersen BK et al. (2010). Gut microbiota in human adults with type 2 diabetes differs from non-diabetic adults. PLoS One 5: e9085.

Le Chatelier E, Nielsen T, Qin J, Prifti E, Hildebrand F, Falony G et al. (2013). Richness of human gut microbiome correlates with metabolic markers. Nature 500: $541-546$.

Li M, Wang B, Zhang M, Rantalainen M, Wang S, Zhou H et al. (2008). Symbiotic gut microbes modulate human metabolic phenotypes. Proc Natl Acad Sci USA 105: 2117-2122.

Liu IX, Durham DG, Richards RM. (2000). Baicalin synergy with beta-lactam antibiotics against methicillinresistant Staphylococcus aureus and other betalactam-resistant strains of $S$. aureus. J Pharm Pharmacol 52: 361-366.

Lozupone C, Knight R. (2005). UniFrac: a new phylogenetic method for comparing microbial communities. Appl Environ Microbiol 71: 8228-8235.

Ludwig W, Strunk O, Westram R, Richter L, Meier H, Yadhukumar et al. (2004). ARB: a software environment for sequence data. Nucleic Acids Res 32: 1363-1371.

Luo CF, Yuan M, Chen MS, Liu SM, Zhu L, Huang BY et al. (2011). Pharmacokinetics, tissue distribution and relative bioavailability of puerarin solid lipid nanoparticles following oral administration. Int J Pharm 410: 138-144. 
Park DY, Ahn YT, Park SH, Huh CS, Yoo SR, Yu R et al. (2013). Supplementation of Lactobacillus curvatus HY7601 and Lactobacillus plantarum KY1032 in diet-induced obese mice is associated with gut microbial changes and reduction in obesity. PLoS One 8: e59470.

Qin J, Li Y, Cai Z, Li S, Zhu J, Zhang F et al. (2012). A metagenome-wide association study of gut microbiota in type 2 diabetes. Nature 490: 55-60.

Rabbani GH, Butler T, Knight J, Sanyal SC, Alam K. (1987). Randomized controlled trial of berberine sulfate therapy for diarrhea due to enterotoxigenic Escherichia coli and Vibrio cholerae. J Infect Dis 155: 979-984.

Rautio M, Saxen H, Siitonen A, Nikku R, Jousimies-Somer H. (2000). Bacteriology of histopathologically defined appendicitis in children. Pediatr Infect Dis $J$ 19: 1078-1083.

Rautio M, Eerola E, Vaisanen-Tunkelrott ML, Molitoris D, Lawson P, Collins MD et al. (2003). Reclassification of Bacteroides putredinis (Weinberg et al., 1937) in a new genus Alistipes gen. nov., as Alistipes putredinis comb. nov., and description of Alistipes finegoldii sp. nov., from human sources. Syst Appl Microbiol 26: 182-188.

Saulnier DM, Riehle K, Mistretta TA, Diaz MA, Mandal D, Raza S et al. (2011). Gastrointestinal microbiome signatures of pediatric patients with irritable bowel syndrome. Gastroenterology 141: 1782-1791.

Shen J, Zhang B, Wei G, Pang X, Wei H, Li M et al. (2006). Molecular profiling of the Clostridium leptum subgroup in human fecal microflora by PCR-denaturing gradient gel electrophoresis and clone library analysis. Appl Environ Microbiol 72: 5232-5238.

Shin NR, Lee JC, Lee HY, Kim MS, Whon TW, Lee MS et al. (2013). An increase in the Akkermansia spp. population induced by metformin treatment improves glucose homeostasis in diet-induced obese mice. Gut 63: 727-735.

Shoelson SE. (2006). Inflammation and insulin resistance. $J$ Clin Invest 116: 1793-1801.

Sokol H, Pigneur B, Watterlot L, Lakhdari O, Bermudez-Humaran LG, Gratadoux JJ et al. (2008). From the cover: Faecalibacterium prausnitzii is an anti-inflammatory commensal bacterium identified by gut microbiota analysis of Crohn disease patients. Proc Natl Acad Sci USA 105: 16731-16736.

Tang J, Feng Y, Tsao S, Wang N, Curtain R, Wang Y. (2009). Berberine and Coptidis rhizoma as novel antineoplastic agents: a review of traditional use and biomedical investigations. J Ethnopharmacol 126: 5-17.

Tian N, Wang J, Wang P, Song X, Yang M, Kong L. (2013). NMR-based metabonomic study of Chinese medicine Gegen Qinlian Decoction as an effective treatment for type 2 diabetes in rats. Metabolomics 9: 1228-1242.

Tong XL, Zhao HL, Lian FM, Zhou Q, Xia L, Zhang JC et al. (2011). Clinical observations on the dose-effect relationship of Gegen Qin Lian Decoction on 54 out-patients with type 2 diabetes. J Traditional Chinese Med 31: 56-59.

Turnbaugh PJ, Backhed F, Fulton L, Gordon JI. (2008). Diet-induced obesity is linked to marked but reversible alterations in the mouse distal gut microbiome. Cell Host Microbe 3: 213-223.

Waisundara VY, Hsu A, Tan BK, Huang D. (2009). Baicalin reduces mitochondrial damage in streptozotocin-induced diabetic Wistar rats. Diabetes Metab Res Rev 25: 671-677.

Wang T, Cai G, Qiu Y, Fei N, Zhang M, Pang X et al. (2011). Structural segregation of gut microbiota between colorectal cancer patients and healthy volunteers. ISME J 6: 320-329.

Whiting DR, Guariguata L, Weil C, Shaw J. (2011). IDF diabetes atlas: global estimates of the prevalence of diabetes for 2011 and 2030. Diabetes Res Clin Pract 94: 311-321.

Xiao S, Fei N, Pang X, Shen J, Wang L, Zhang B et al. (2013). A gut microbiota-targeted dietary intervention for amelioration of chronic inflammation underlying metabolic syndrome. FEMS Microbiol Ecol 87: 357-367.

$\mathrm{Xu} \mathrm{Y,} \mathrm{Wang} \mathrm{L,} \mathrm{He} \mathrm{J,} \mathrm{Bi} \mathrm{Y,} \mathrm{Li} \mathrm{M,} \mathrm{Wang} \mathrm{T} \mathrm{et} \mathrm{al.} \mathrm{(2013).}$ Prevalence and control of diabetes in Chinese adults. JAMA 310: 948-959.

Zhang C, Zhang M, Wang S, Han R, Cao Y, Hua W et al. (2009). Interactions between gut microbiota, host genetics and diet relevant to development of metabolic syndromes in mice. ISME J 4: 232-241.

Zhang C, Zhang M, Pang X, Zhao Y, Wang L, Zhao L. (2012a). Structural resilience of the gut microbiota in adult mice under high-fat dietary perturbations. ISME J 6: 1848-1857.

Zhang CH, Xu GL, Liu YH, Rao Y, Yu RY, Zhang ZW et al. (2013). Anti-diabetic activities of Gegen Qinlian Decoction in high-fat diet combined with streptozotocininduced diabetic rats and in 3T3-L1 adipocytes. Phytomedicine 20: 221-229.

Zhang X, Zhao Y, Zhang M, Pang X, Xu J, Kang C et al. (2012b). Structural changes of gut microbiota during berberine-mediated prevention of obesity and insulin resistance in high-fat diet-fed rats. PLoS One 7: e42529.

Zhang Y, Li X, Zou D, Liu W, Yang J, Zhu N et al. (2008). Treatment of type 2 diabetes and dyslipidemia with the natural plant alkaloid berberine. J Clin Endocrinol Metab 93: 2559-2565.

Zhao L. (2013). The gut microbiota and obesity: from correlation to causality. Nat Rev Microbiol 11: 639-647.

Zhu XY, Su GY, Li ZH, Yue TL, Yan XZ, Wei HL. (1979). The metabolic fate of the effective components of puerariae. III. The metabolism of puerarin [article in Chinese]. Acta Pharm Sin 14: 349-355.

(c) (1) $\odot \Theta$ This work is licensed under a Creative (1) Commons Attribution-NonCommercialNoDerivs 3.0 Unported License. The images or other third party material in this article are included in the article's Creative Commons license, unless indicated otherwise in the credit line; if the material is not included under the Creative Commons license, users will need to obtain permission from the license holder to reproduce the material. To view a copy of this license, visit http://creativecommons.org/ licenses/by-nc-nd/3.0/

Supplementary Information accompanies this paper on The ISME Journal website (http://www.nature.com/ismej) 